\title{
Behavioral outcomes of picky eating in childhood: a prospective study in the general population
}

\author{
Sebastian Cardona Cano, ${ }^{1,2}$ Hans W. Hoek, ${ }^{2,3,4}$ Daphne van Hoeken, ${ }^{2}$ Lisanne M. de \\ Barse, ${ }^{1,5}$ Vincent W.V. Jaddoe, ${ }^{1,5,6}$ Frank C. Verhulst, ${ }^{1,7}$ and Henning Tiemeier ${ }^{5,7,8}$ \\ ${ }^{1}$ The Generation R Study Group, Erasmus University Medical Center, Rotterdam; ${ }^{2}$ Parnassia Psychiatric Institute, \\ The Hague, The Netherlands; ${ }^{3}$ Department of Epidemiology, Mailman School of Public Health, Columbia University, \\ New York, NY, USA; ${ }^{4}$ Department of Psychiatry, University Medical Center Groningen, University of Groningen, \\ Groningen; ${ }^{5}$ Department of Epidemiology, Erasmus University Medical Center, Rotterdam; ${ }^{6}$ Department of \\ Pediatrics, Erasmus University Medical Center - Sophia Children's Hospital, Rotterdam; ${ }^{7}$ Department of Child and \\ Adolescent Psychiatry/Psychology, Erasmus University Medical Center - Sophia Children's Hospital, Rotterdam; \\ ${ }^{8}$ Department of Psychiatry, Erasmus University Medical Center, Rotterdam, The Netherlands
}

\begin{abstract}
Background: Picky eaters in the general population form a heterogeneous group. It is important to differentiate between children with transient picky eating (PE) and persistent PE behavior when adverse outcomes are studied. We analyzed four PE trajectories to determine the associations with child mental health prospectively. Methods: From a population-based cohort, 3,748 participants were assessed for $\mathrm{PE}$ at 1.5, 3, and 6 years of age using maternal reports. Four trajectories were defined: persistent (PE at all ages); remitting (PE before 6 years only); late-onset (PE at 6 years only); and never (no PE at any assessment). Child's problem behaviors were assessed with the Teacher's Report Form at 7 years of age. We examined associations between picky eating trajectories and emotional problems, behavioral problems and pervasive developmental problems using logistic regressions. Analyses were adjusted for child, parental, and socioeconomic confounders. We also adjusted for maternal-reported baseline problem behavior at age 1.5 years; the never picky eating group was used as reference. Results: Persisting PE predicted pervasive developmental problems at age 7 years $(\mathrm{OR}=2.00,95 \% \mathrm{CI}: 1.10-3.63)$. The association remained when adjusted for baseline pervasive developmental problems at 1.5 years $(\mathrm{OR}=1.96,95 \% \mathrm{CI}: 1.10-3.51)$. Persistent $\mathrm{PE}$ was not associated with behavioral ( $\mathrm{OR}=0.92,95 \% \mathrm{CI}$ : 0.53-1.60) or emotional problems $(\mathrm{OR}=1.24,95 \% \mathrm{CI}: 0.74-2.07)$. Other PE trajectories were not related to child behavioral or emotional problems. Conclusions: Persistent PE may be a symptom or sign of pervasive developmental problems, but is not predictive of other behavioral problems. Remitting PE was not associated with adverse mental health outcomes, which further indicates that it may be part of normal development. Keywords: Picky eating; emotional problems; behavioral problems; pervasive developmental problems.
\end{abstract}

\section{Introduction}

Picky eating is a frequent eating problem in early childhood, characterized by food refusal, eating a limited variety of food, an unwillingness to try new food (food neophobia) (Dovey, Staples, Gibson, \& Halford, 2008) and aberrant eating behaviors, such as low enjoyment of food, slowness in eating and higher satiety responsiveness (Cardona Cano, Hoek, $\&$ Bryant-Waugh, 2015a). The prevalence of picky eating is highest $(14-50 \%)$ in preschool children (Carruth, Ziegler, Gordon, \& Barr, 2004; Dovey et al., 2008; Mascola, Bryson, \& Agras, 2010), and declines (7-27\%) in later childhood (Mascola et al., 2010; Micali et al., 2011a). Incidence also declines after preschool age (Mascola et al., 2010). The high prevalence and incidence are an indication that picky eating in the preschool age is often part of normal development (Mascola et al., 2010). Indeed many health professionals tend to regard picky eating as a normal phase which eventually passes (Nicholls, Christie, Randall, \& Lask, 2001). However, this is in contrast to how many parents experience

Conflict of interest statement: No conflicts declared. picky eating, that is, as a major cause of concern (Cerro, Zeunert, Simmer, \& Daniels, 2002; Goh \& Jacob, 2012; Mascola et al., 2010; Wright, Parkinson, Shipton, \& Drewett, 2007). Parents often seek medical help for their child's picky eating (Wright et al., 2007), and express frustration with physicians for dismissing their concerns (McKee, Maher, Deen, \& Blank, 2010). Our previous report within the Generation R Study on picky eating trajectories, confirmed that the majority of children's picky eating problems in the preschool age remitted before the age of 6 years (Cardona Cano et al., 2015b). However, we also found a small group of children with persisting picky eating problems who had a lower birth weight, were more often male and from a nonDutch and low socioeconomic background, compared with nonpicky eaters (Cardona Cano et al., 2015b).

In previous studies, picky eating was associated with higher levels of behavioral, emotional, and pervasive developmental problems in childhood (Jacobi, Schmitz, \& Agras, 2008; Micali et al., 2011a; Nicholls et al., 2001) and was suggested to be a precursor for anorexia nervosa (Marchi \& Cohen, 1990). The most recent studies concluded 
that picky eating in children of school age must be seen as a risk factor or marker for general psychopathology, rather than a precursor of eating disorders (Jacobi et al., 2008; Micali et al., 2011a). However, picky eating problems are also specifically associated with pervasive developmental disorders (Bandini et al., 2010). The prevalence of picky eating in children with autism was found to be as high as 90\% (Sharp et al., 2013) and often present from early age onwards (Emond, Emmett, Steer, \& Golding, 2010). In addition, feeding problems and eating disorders are associated with anxiety problems (Galloway, Lee, \& Birch, 2003; Swinbourne \& Touyz, 2007), and distorted child-parent interactions are suggested to play an important role in feeding problems (Davies et al., 2006).

However, most picky eating studies have some important limitations. First, most studies were limited by their cross-sectional design. Second, they did not differentiate between different trajectories, clustering remitting and persistent picky eaters. Third, a lack of correction for baseline differences makes temporal inferences difficult. Also, most studies did not adjust or only poorly adjusted for confounders. Only a few studies included child, parental, and socioeconomic characteristics (Cardona Cano et al., 2015b; Hafstad, Abebe, Torgersen, \& von Soest, 2013; Jacobi et al., 2008). Gender, weight at birth, parental income, maternal ethnicity and age, birth order, higher levels of child emotionality and maternal negative affectivity were found to predict picky eating at later age (Cardona Cano et al., 2015b; Hafstad et al., 2013). Lastly, the majority of studies in the field of eating disorders research rely on one informant to report both the determinant and outcome. However, this practice of using a single informant can lead to spurious associations, that is, information bias (shared method variance) (Ringoot et al., 2015). Typically, mothers' reports are used to assess picky eating as well as emotional and behavioral problems, possibly introducing this type of bias.

It is important to study the course and outcome of picky eating in the general population to determine which children are at high risk for adverse mental health outcomes. Furthermore, this should be evaluated in the context of the child's age. First, we hypothesize that remitting picky eating problems in the preschool age (0-4 years) are part of normal development and are not associated with an increased risk of any adverse mental health problems. Second, we hypothesize that children with persisting picky eating problems have a higher risk for adverse mental health outcomes. In particular, we expect that persistent picky eating is associated with more pervasive developmental problems and anxiety problems. Third, we will test whether lateonset picky eating is associated with emotional or behavioral problems; however, there are insufficient studies to date to formulate a specific hypothesis for this item.

\section{Methods \\ Study design and population}

This study was embedded within the Generation R Study (Jaddoe et al., 2012). The Generation R Study is a prospective population-based cohort in Rotterdam (the Netherlands), that aims to identify environmental and genetic causes of normal and abnormal growth, development, and health from fetal life onward. Pregnant women residing in Rotterdam with an expected delivery date between April 2002 and January 2006 were invited to participate. Written informed consent was obtained from all participants. The Medical Ethical Committee of the Erasmus Medical Center, Rotterdam, approved the study. Information about child and family characteristics was obtained by postal questionnaires filled out by parents, and from the medical records of hospitals, midwives, and community Child Health Centers.

Picky eating was assessed by parental report questionnaires when children were $1.5,3$, and 6 years old. Children who were not assessed for picky eating at any of these time points, or with an inconsistent picky eating pattern, were excluded from the study. Behavioral outcomes were determined using the Teacher Report Form (TRF) when the child was 7 years old (mean 6.7, SD 1.3 years; $N=4,696)$. A total of 3,748 $(78.8 \%)$ children were included in the present study.

\section{Measures}

Trajectories of picky eating. Picky eating was assessed with two questions of the Child Behavioral Checklist (CBCL) at age $1.5,3$, and 6 years. The detailed methodology is described elsewhere (Cardona Cano et al., 2015b). In short: at each assessment wave, mothers indicated whether their child 'did not eat well' and 'refused to eat' on a 3-point Likert scale. Based on the sum score of these two items (sum range 2-6) children with a score of sometimes and/or often (score of $\geq 4$ ) were identified as a picky eater. This method approximates the concept of picky eating as defined by Dovey et al. (2008), with reduced caloric intake, lower variety of foods, higher food fussiness, less enjoyment of food, higher satiety response, and slowness in eating (Cardona Cano et al., 2015b). It is important to note that our method aims to determine picky eating problems in the general population, including (but not limited to) clinically significant 'picky eating disorders'. Four main picky eating trajectory groups were created: never picky eaters - those who were never identified as picky eaters; remitting picky eaters - those who were picky eaters at 1.5 and/or 3 years, but not at 6 years of age; late-onset picky eaters those who were picky eaters at 6 years of age only; and persistent picky eaters - those who were picky eaters during all assessment waves $(1.5,3$, and 6 years). The remaining 242 children with an inconsistent pattern (i.e., children assessed as picky eaters at 1.5 and 6 years, but not at 3 years, and children that were picky eaters at 3 and 6 years, but not at 1.5 years) were excluded from further analysis for two main reasons: the accurate categorization would depend strongly on future follow-up of picky eating status with the possibility that these children would then be categorized into remitting, lateonset, or persistent picky eaters; and analyses of this group revealed a different pattern compared to the other trajectories and did not differ from the never picky eaters (results not shown).

Child's problem behavior. To determine children's problem behavior the Dutch translation of the TRF (Verhulst, van Ende, \& Koot, 1997) was used. The TRF is the teacher version of the CBCL, comprising 120 problem items that can be scored on a 3-point Likert scale (i.e., not true, sometimes true, or often true). The TRF has the following six DSM-Oriented Scales: affective problems, anxiety problems, pervasive 
developmental problems, attention deficit hyperactivity problems, oppositional defiant problems, and conduct problems. The DSM-Oriented Scale problems were defined using the established borderline clinical cut-offs (Achenbach \& Rescorla, 2001); however, linear regression analyses with continuously modeled outcomes are also presented to demonstrate that our findings do not depend on choice of cut-off (see Table S1) Three main groups of problems were formed in line with Mical et al. (2011a): 'emotional problems' consisting of the summed anxiety and affective problems; 'behavioral problems' consisting of attention hyperactivity and oppositional defiant problems; and pervasive developmental problems. Conduct problems were excluded from the behavioral problem group since, at a young age, the diagnosis of conduct disorder has a low prevalence (Ford, Goodman, \& Meltzer, 2003). The Dutch TRF has good reliability and validity (Verhulst et al., 1997).

Baseline problem level was assessed by the mother using the CBCL/ 1.5-5 when the child was 1.5 years of age. The CBCL (Tick, van der Ende, \& Verhulst, 2007) is a 99-item parent report questionnaire that assesses child emotional and behavioral problems in a manner similar to the TRF. The Dutch CBCL is reported to have good reliability and validity (Tick et al., 2007). Three main problem groups (as described above) were formed. However, in contrast to the TRF, the CBCL scale scores were used continuously with a higher score indicating more problems. One of the items used to assess picky eating was also present in the emotional problems scale. To avoid bias, this item was excluded from the emotional problems scale score.

Child, parental, and sociodemographic information. Based on previous studies (Cardona Cano et al., 2015b; Dubois, Farmer, Girard, Peterson, \& Tatone-Tokuda, 2007; Hafstad et al., 2013; Micali, Simonoff, Stahl, \& Treasure, 2011b), we defined several child, family, and socioeconomic characteristics as confounders. Information about child gender, birth weight, and gestational age at birth was obtained from midwife and hospital registries. Maternal ethnicity, family income, and child's birth order were assessed by postal questionnaire. Maternal educational level was coded as high (some college or university education), middle (secondary education), or low (primary education or none). Family income per month was coded as high or middle (above median income $>2200$ euro), low (1200-2200 euro), or very low (<1200 euro). Birth order was defined as firstborn or later born. Maternal ethnicity was coded as Dutch, Moroccan, Turkish, a combined code (Sur/Ant/Cape) for mothers with a Surinamese, Dutch Antillean or Cape Verdian ethnicity, other Western, and other non-Western. Birth weight is given in grams, gestational age at birth in weeks, and BMI in bodyweight $/$ height ${ }^{2}\left(\mathrm{~kg} / \mathrm{m}^{2}\right)$.

Maternal psychiatric symptoms were assessed with the Brief Symptom Inventory (BSI) during pregnancy. The BSI is a validated self-report questionnaire (de Beurs \& Zitman, 2006 Derogatis \& Melisaratos, 1983) that consists of 53 items scored on a 5-point Likert scale. It assesses a spectrum of psychiatric problems such as anxiety, depression, somatization, and hostility problems. The global severity index is the mean of all subscales and is an appropriate measure of general psychopathology (Derogatis \& Melisaratos, 1983; Skeem et al., 2006); that overall mean score was used as a continuous measure, with higher scores indicating more problems.

\section{Statistical analysis}

To examine the relationship between picky eating and child's behavioral problems separate logistic regressions were carried out with emotional problems, behavioral problems, and pervasive developmental problems as outcome, and the trajectories of picky eating as the independent variable. The never picky eating group was used as the reference group. First
(Model 1), a univariate logistic regression was performed. In the second analysis (Model 2) many of the confounder variables found to predict picky eating (Cardona Cano et al., 2015b; Hafstad et al., 2013) were added. These included gender, weight at birth, maternal ethnicity and income, birth order and maternal psychopathology. Because maternal age did not predict picky eating in our earlier study (Cardona Cano et al., 2015b), it was not included as a confounder in the present study. Finally (Model 3), to address the temporal sequence of the relation, we corrected for baseline child behavioral problems. For this, we also adjusted for maternalreported child behavioral problem using the $\mathrm{CBCL}$ at age 1.5 years. In addition, we reran all analyses using maternalreported emotional, behavioral, and pervasive developmental outcomes using the CBCL at 6 years of age, to enable comparison with other studies. These additional analyses are presented in Table S2 and are contrasted with analyses using teacher reports in the same sample and also highlight possible informant bias. Except for the dependent variables, missing values were estimated using multiple imputation techniques. As the CBCL data included some missing values $(<30 \%$ per assessment wave), proportions of trajectories of picky eating were based on multiple imputation if one or more scores were obtained. The pervasive developmental problems group was the only dependent variable (outcome) with missing data $(N=3734$, missings $n=14)$. The presented results are based on pooled estimates of five imputed datasets. Analyses were performed using STATA/SE 12.0.

\section{Results Study population}

General child and family characteristics of the study population are presented in Table 1 . The amount of boys and girls was almost equal. The majority of the mothers were of Dutch ethnicity (57.9\%), and from a higher socioeconomic status $156.5 \%$ higher education). The majority of the children never had picky eating problems $(51.4 \% ; n=1926)$. Approximately $5.5 \%(n=206)$ were persistent picky eaters, while $31.9 \%(n=1197)$ were remitting picky eaters. These numbers are best estimates (variation $<4 \%$ of sample) as they are based on imputed data.

\section{Picky eating trajectories and associations with child behavioral problems}

No associations were found between remitting, lateonset, persistent picky eaters and never picky eaters, and behavioral problems (Table 2).

In the unadjusted analyses (Model 1), remitting and persistent picky eaters showed more emotional problems than the reference group (Table 3). No difference in emotional problems was found between late-onset picky eaters and never picky eaters. After adjusting for child, family, and sociodemographic variables (Model 2), no differences in emotional problems were found.

Persistent picky eating was associated with more pervasive developmental problems, unadjusted (Model 1; OR $=2.41,9 \% \mathrm{CI}: 1.37-4.22$ ), and after adjusting for confounders (Model 2; adjusted $\mathrm{OR}=2.00, \quad 95 \%$ CI: 1.10-3.63) $\quad$ (Table 4). After 
Table 1 Population characteristics

\begin{tabular}{|c|c|}
\hline & $N=3,748$ \\
\hline \multicolumn{2}{|l|}{ Child characteristic } \\
\hline \multicolumn{2}{|l|}{ Gender } \\
\hline Boy, \% & 50.6 \\
\hline Girl, \% & 49.4 \\
\hline \multicolumn{2}{|l|}{ Birth weight } \\
\hline Normal weight, \% & 88.6 \\
\hline Underweight, \% & 4.3 \\
\hline Overweight, \% & 7.2 \\
\hline \multicolumn{2}{|l|}{ Gestational age at birth } \\
\hline Aterm, \% & 87.5 \\
\hline Preterm, \% & 4.9 \\
\hline Postterm, \% & 7.6 \\
\hline \multicolumn{2}{|l|}{ Parental characteristic } \\
\hline Age mother ${ }^{a}$, in years & $30.9(5.0)$ \\
\hline \multicolumn{2}{|l|}{ Mean (SD) } \\
\hline Age father ${ }^{a}$, in years & $33.7(5.7)$ \\
\hline \multicolumn{2}{|l|}{ Mean (SD) } \\
\hline \multicolumn{2}{|l|}{ Maternal ethnicity } \\
\hline Dutch, \% & 57.9 \\
\hline Moroccan, \% & 5.5 \\
\hline Turkish, \% & 8.5 \\
\hline Sur/Ant/Cape ${ }^{\mathrm{b}}, \%$ & 12.3 \\
\hline Other Western, \% & 8.1 \\
\hline Other non-Western, \% & 7.7 \\
\hline \multicolumn{2}{|l|}{ Maternal educational level ${ }^{\mathrm{c}}$} \\
\hline $\operatorname{High}^{\mathrm{d}}, \%$ & 56.5 \\
\hline Middle, \% & 38.8 \\
\hline Low, \% & 4.7 \\
\hline \multicolumn{2}{|l|}{ Family income } \\
\hline High or middle, $\%$ & 59.6 \\
\hline Low, \% & 15.3 \\
\hline Very low, \% & 25.1 \\
\hline \multicolumn{2}{|l|}{ Birth order } \\
\hline Firstborn, \% & 55.15 \\
\hline Later born, \% & 44.85 \\
\hline \multicolumn{2}{|l|}{ Smoking during pregnancy } \\
\hline No, \% & 75.7 \\
\hline Stopped at pregnancy, \% & 8.8 \\
\hline Yes, \% & 15.5 \\
\hline \multicolumn{2}{|l|}{ Picky Eating Trajectories ${ }^{\mathrm{e}}$} \\
\hline Never & 1,926 \\
\hline Remitting & 1,197 \\
\hline Late-onset & 177 \\
\hline Persistent & 206 \\
\hline
\end{tabular}

${ }^{\text {a At intake }}$

${ }^{\mathrm{b}}$ Suriname / Antillean / Cape Verdean

${ }^{\mathrm{c}}$ Highest followed

${ }^{\mathrm{d}}$ Low $=$ none $\quad$ or $\quad$ primary, middle $=$ secondary school, high $=$ higher vocational education/ university

${ }^{\mathrm{e}} n$ is based on imputed trajectory groups. The inconsistent group ( $n=242)$ was excluded.

additionally adjusting for baseline pervasive developmental problems at 1.5 years, persistent picky eating remained associated with a higher risk of pervasive developmental problems (Model 3; adjusted OR $=1.96,95 \%$ CI: $1.10-3.51$; data not in table). None of the other picky eating trajectories were associated with pervasive developmental problems.

\section{Discussion}

In this population-based study, we found that persistent picky eating was longitudinally associated
Table 2 Longitudinal association ${ }^{\mathrm{a}}$ between trajectories of picky eaters and borderline behavioral problems

\begin{tabular}{lrll}
\hline & & \multicolumn{2}{c}{ Behavioral problems } \\
\cline { 3 - 4 } $\begin{array}{l}\text { Picky eating } \\
\text { trajectories }\end{array}$ & \multicolumn{1}{c}{$N^{\mathrm{b}}$} & \multicolumn{1}{c}{$\begin{array}{c}\text { Model 1 } \\
\text { OR }(95 \% \mathrm{CI})\end{array}$} & $\begin{array}{c}\text { Model } 2 \\
\text { OR }(95 \% \mathrm{CI})\end{array}$ \\
\hline Never & 1,926 & Reference & Reference \\
Remitting & 1,197 & $1.07(0.74-1.55)$ & $1.03(0.66-1.61)$ \\
Late-onset & 177 & $1.42(0.64-3.13)$ & $1.05(0.42-2.61)$ \\
Persistent & 206 & $0.92(0.53-1.60)$ & $0.66(0.38-1.15)$ \\
\hline
\end{tabular}

Behavioral problems; attention-deficit hyperactivity and oppositional defiant problems.

Model 2: adjusted gender, weight at birth, gestational age at birth, maternal ethnicity, household income, birth order, and maternal psychopathology.

${ }^{a}$ Logistic regression.

${ }^{\mathrm{b}} \mathrm{N}$ is based on imputed trajectory groups. The inconsistent group $(n=242)$ was excluded.

Table 3 Longitudinal association ${ }^{\mathrm{a}}$ between trajectories of picky eaters and borderline emotional problems

\begin{tabular}{lrcl}
\hline & & \multicolumn{2}{c}{ Emotional problems } \\
\cline { 3 - 4 } $\begin{array}{l}\text { Picky eating } \\
\text { trajectories }\end{array}$ & \multirow{2}{*}{$N^{\mathrm{b}}$} & Model 1 & \multicolumn{1}{c}{ Model 2} \\
OR $(95 \% \mathrm{CI})$ & OR $(95 \% \mathrm{CI})$ \\
\hline Never & 1,926 & Reference & Reference \\
Remitting & 1,197 & $\mathbf{1 . 5 4 *} \mathbf{( 1 . 0 1 - 2 . 3 6 )}$ & $1.47(0.92-2.33)$ \\
Late-onset & 177 & $1.53(0.67-3.51)$ & $1.21(0.56-2.64)$ \\
Persistent & 206 & $\mathbf{1 . 7 1 * ( 1 . 0 1 - 2 . 9 1 )}$ & $1.24(0.74-2.07)$ \\
\hline
\end{tabular}

Emotional problems; anxiety and affective problems.

Model 2: adjusted gender, weight at birth, gestational age at birth, maternal ethnicity, household income, birth order, and maternal psychopathology.

The bold values are given to accentuate that these values are significant findings.

${ }^{a}$ Logistic regression.

${ }^{\mathrm{b}} N$ is based on imputed trajectory groups. The inconsistent group $(n=242)$ was excluded.

$* p<.05$.

with pervasive developmental problems at age 7 years as reported by teachers, even after adjustment of baseline pervasive developmental problems at 1.5 years. Remitting and late-onset picky eating were not associated with adverse mental health outcomes.

In line with our first hypothesis, this study demonstrates that remitting picky eating was not prospectively associated with adverse mental health outcomes. This suggests that remitting picky eating in preschool children can be seen as part of normal development in the general population (Nicholls et al., 2001); a behavior that might be considered as age-appropriate and will eventually remit without behavioral or emotional consequences. This is further strengthened by the fact that our findings are based on a longitudinal design. However, in the present study we did not include somatic health measures and other adverse outcomes cannot be ruled out (Drewett, Corbett, \& Wright, 2006; Wright et al., 2007). 
Table 4 Longitudinal association ${ }^{\mathrm{a}}$ between trajectories of picky eaters and borderline pervasive developmental problems

\begin{tabular}{|c|c|c|c|}
\hline \multirow{2}{*}{$\begin{array}{l}\text { Picky } \\
\text { eating } \\
\text { trajectories }\end{array}$} & \multirow[b]{2}{*}{$N^{\mathrm{b}}$} & \multicolumn{2}{|c|}{ Pervasive developmental problems } \\
\hline & & $\begin{array}{c}\text { Model } 1 \\
\text { OR (95\% CI) }\end{array}$ & $\begin{array}{c}\text { Model } 2 \\
\text { OR (95\% CI) }\end{array}$ \\
\hline Never & 1,920 & Ref & Ref \\
\hline Remitting & 1,192 & $1.02(0.64-1.61)$ & $0.97(0.59-1.59)$ \\
\hline Late-onset & 176 & $0.70(0.21-2.24)$ & $0.57(0.16-2.05)$ \\
\hline Persistent & 205 & $2.41 * *(1.37-4.22)$ & $2.00 *(1.10-3.63)$ \\
\hline
\end{tabular}

Model 2: adjusted gender, weight at birth, gestational age at birth, maternal ethnicity, household income, birth order, and maternal psychopathology.

The bold values are given to accentuate that these values are significant findings.

${ }^{a}$ Logistic Regression.

${ }^{\mathrm{b}} N$ is based on imputed trajectory groups. The inconsistent group $(n=241)$ was excluded.

$* p<.05 ; * *<<.01$.

Second, we hypothesized that persistent picky eating would be prospectively associated with pervasive developmental problems. In line with clinical studies reporting more picky eating problems among children with autism spectrum disorders (ASD) (Bandini et al., 2010), our study suggests that persistent picky eating is also more common in children from the general population with elevated pervasive developmental problems. Importantly, when we corrected for baseline pervasive developmental problems, persistent picky eating remained related to pervasive developmental problems at age 7 years. Thus, the finding cannot be explained by developmental problems early in life. This assessment was based on maternal reports, as parents usually recognize signs of autism in an early stage (Ozonoff et al., 2009). Potentially, picky eating can help to detect pervasive developmental problems earlier, as picky eating in young children is easily noticed by parents. In the study of Emond et al. (2010), parents reported that difficulty in eating is often present in children with autism from infancy (6 months) onwards and persists throughout early childhood; our finding that persistent picky eating can be an early symptom or sign for pervasive developmental problems extends this observation and suggests that in the general population picky eating can precede other pervasive developmental problems symptoms.

However, the median age of the first ASD diagnosis remains older than age 4 years (Centers for Disease Control and Prevention, 2014; Maenner et al., 2013). Persistent picky eating trajectories in our study are based on assessments from 1.5 to 6 years of age, thus a majority of children with ASD would already be diagnosed before persistent picky eating can be defined. However, as the age at which ASD is diagnosed is inversely associated with the number of symptoms observed (Maenner et al., 2013), persisting picky eating can be used to detect ASD in a minority of children in those with less severe or clear symptoms. Future studies are needed to evaluate if a persistent picky eating trajectory can be delineated earlier, that is at age 4-5 years. Since parents often seek medical help for their child's eating behavior (Wright et al., 2007), clinicians should pay attention to children who persist in having picky eating behavior, as these children are at higher risk of pervasive developmental disorders. Some caution is warranted as the CBCL assesses pervasive developmental problems and is not a diagnostic instrument; however, several studies have demonstrated that the CBCL pervasive developmental problem scale can be used to screen for ASD, but has a particularly high specificity in the assessment of pervasive developmental disorders (Ooi, Rescorla, Ang, Woo, \& Fung, 2011; Sikora, Hall, Hartley, Gerrard-Morris, \& Cagle, 2008; So et al., 2013).

We did not confirm our hypothesis that persistent picky eating was also prospectively associated with anxiety problems. Rather, persistent picky eating was not associated with problems other than pervasive developmental problems. This is in contrast with an earlier report of the ALSPAC study that found strong associations of picky eating with behavioral and emotional problems (Micali et al., 2011b). In our study, the existing association between persistent picky eating and emotional problems disappeared when confounders were controlled for. Also, the present study found lower odds ratios compared with Micali et al. in the UK (2011b). The differences between the two studies might be explained by the design of our study (cross-sectional vs. longitudinal and repeated measures design) and, most importantly by a different informant (a teacher report vs. a mother report) as a measure for outcome. An earlier study showed that, when maternal reports are used for both the determinant and the outcome measure, the associations were strongly inflated (Ringoot et al., 2015). Thus, when mothers report both picky eating and problem behavior of the child, any observed association of picky eating with behavior and emotional problems is prone to reporter bias. Furthermore, mothers who are overconcerned about their child's wellbeing might rate their child's behavior as problematic in general. Our results suggest that the associations of picky eating with emotional and behavior problems may be inflated when mothers' reports of emotional and behavior problems are used (see Table S2). However, others may argue that teachers underreport (which would probably reduce precision) or more often incorrectly report, which would reduce the estimated effect of the associations.

Some caution is required when interpreting these results. First, because the concept of picky eating has not been fully operationalized (Dovey et al., 2008) and the boundaries between picky eating, food neophobia and eating disorders are not yet well defined. Also, in the present study, we defined trajectories of picky eating to differentiate subgroups across time that 
might have distinct outcomes. Although we found no association between picky eating and emotional problems, picky eating persisting from early to late childhood might predict eating disorders in adolescence (Marchi \& Cohen, 1990) or might be a risk factor for other severe psychopathology. However, this was beyond the scope of the present study and more research is required on this topic.

Our results emphasize the importance of differentiating between trajectories of picky eaters, as picky eating comprises distinct groups with different symptom clusters ranging from mild symptoms to clinical disorders such as Avoidant/Restrictive Food Intake Disorder (ARFID). ARFID can be considered an extreme form of picky eating and is associated with more pervasive developmental disorders compared with other eating disorders in a clinical setting (Nicely, Lane-Loney, Masciulli, Hollenbeak, \& Ornstein, 2014). Therefore, we cautiously speculate that persistent picky eaters are at a higher risk for the development of ARFID.

Finally, we tested whether late-onset picky eating was associated with emotional or behavioral problems. Although late-onset picky eating was not longitudinally associated with any adverse mental health outcome, a study by Micali et al. (2011a) found more emotional, behavioral, and pervasive problems, as described above. It is possible that our study was underpowered to detect minor differences, given the relatively small group of late-onset picky eating to find differences when comparing them to children without picky eating problems. In the present study, late-onset picky eaters tended to have more emotional and behavioral problems, but only in the unadjusted models; after correcting for confounders the odds ratios were strongly attenuated. This implies that the observed effect of picky eating behavior in early childhood is partially explained by socioeconomic differences between groups.

\section{Strengths and limitations}

This study had several strengths including the large sample size, its population-based longitudinal design and inclusion of a large amount of confounders. Additional strengths are the use of the teacher report (as an independent measurement for child psychopathology) and correction for baseline problems.

Some limitations should also be discussed. First, the TRF reports on the DSM-Oriented Scales and is not equivalent to a DSM diagnosis. Thus, some caution is necessary interpreting these results, more so as the borderline clinical cut-off was used. Second, we had no measurements at 4 and 5 years of age in order to better determine picky eaters with a persistent pattern or late-onset. Also, parents might have adjusted eating regimes to compensate for their child's pickiness (Farrow \& Blissett, 2006), resulting in a misclassification of the remitting group. However, maternal reports for the assessment of picky eating have been validated (Carruth \& Skinner, 2000). We used 'my child refuses to eat' and 'my child doesn't eat well' to assess picky eating status. However, previous analyses (Cardona Cano et al., 2015b) found that this method correlates well with measures of picky eating, including a lower variety of food, lower caloric intake, more food fussiness, slowness in eating, and lower enjoyment of food. This indicates that our definition is a valid approximation of the concept. Lastly, a small group of picky eaters was excluded from further analysis due to having an inconsistent picky eating pattern. Follow-up of this group is needed to determine whether children in this group should be classified as remitting or persistent picky eaters, and whether picky eating is associated with adverse mental health outcomes.

\section{Clinical implications}

Persistent picky eating was found to be an early symptom for pervasive developmental problems, whereas remitting picky eating was not associated with adverse mental health outcomes. We cautiously propose to regard remitting picky eating as part of normal development and, in line with consensusbased professional health guidelines, suggest a watchful waiting approach to picky eating problems in preschool age. However, health professionals should be aware of the possible mental health implications of persisting picky eating and, if necessary, perform additional testing.

\section{Supporting information}

Additional Supporting Information may be found in the online version of this article:

Table S1. Associations between trajectories of picky eaters and continuously modeled child's behavioral problems.

Table S2. Possible informant bias in the association between trajectories of picky eating and problem behavior: using teacher versus mother-reported outcome.

\section{Acknowledgements}

The Generation R Study is conducted by the Erasmus University Medical Center Rotterdam in close collaboration with the Erasmus University Rotterdam, School of Law and Faculty of Social Sciences; the Municipal Health Service Rotterdam area, Rotterdam; the Rotterdam Homecare Foundation, Rotterdam; and the Stichting Trombosedienst \& Artsenlaboratorium Rijnmond (STAR), Rotterdam. We gratefully acknowledge the contribution of the participating pregnant women and their partners, general practitioners, hospitals, midwives, and pharmacies in Rotterdam.

The authors declare that they have no conflicts of interest in relation to this work; L.B. works in ErasmusAGE, a center for aging research across the 
life course funded by Nestlé Nutrition (Nestec Ltd.), Metagenics Inc. and AXA. The funders had no role in the design or conduct of this study; collection, management, analysis, or interpretation of the data; and preparation, review, or approval of the manuscript.

\section{Correspondence}

Henning Tiemeier, Department of Child and Adolescent Psychiatry and Psychology, Erasmus Medical Center, P.O. Box 2060, 3000 CB Rotterdam, The Netherlands; Email: h.tiemeier@erasmusmc.nl

\section{Key points}

- Picky eating is a major concern for many parents, although picky eating problems between 1 and 4 years generally remit. Child outcome studies are needed to evaluate the prognosis of persistent and remitting picky eaters.

- Previous studies associated picky eating with more behavioral, emotional, and pervasive developmental problems in childhood, and characterized it as a symptom for general psychopathology.

- In this study, persistent picky eating, but not late-onset or remitting picky eating, was an early sign for pervasive developmental problems.

- Remitting picky eating was not associated with child behavior problems, suggesting that remitting picky eating is part of normal development.

\section{References}

Achenbach, T.M., \& Rescorla, L.A. (2001). Manual for the ASEBA school-age forms \& profiles. Burlington, VT: University of Vermont, Research Center for Children, Youth, \& Families.

Bandini, L.G., Anderson, S.E., Curtin, C., Cermak, S., Evans, E.W., Scampini, R., .. \& \& Must, A. (2010). Food selectivity in children with autism spectrum disorders and typically developing children. Journal of Pediatrics, 157, 259-264.

de Beurs, E., \& Zitman, F.G. (2006). The Brief Symptom Inventory (BSI): The reliability and validity of a brief alternative of the SCL-90. Maandblad Geestelijke Volksgezondheid, 61, 120-137.

Cardona Cano, S., Hoek, H.W., \& Bryant-Waugh, R. (2015a). Picky eating: The current state of research. Current Opinion in Psychiatry, 28, 448-454.

Cardona Cano, S., Tiemeier, H., van Hoeken, D., Tharner, A., Jaddoe, V.W., Hofman, A., ․ \& Hoek, H.W. (2015b). Trajectories of picky eating during childhood: A general population study. International Journal of Eating Disorders, $48,570-579$.

Carruth, B.R., \& Skinner, J.D. (2000). Revisiting the picky eater phenomenon: Neophobic behaviors of young children. Journal of the American College of Nutrition, 19, 771-780.

Carruth, B.R., Ziegler, P.J., Gordon, A., \& Barr, S.I. (2004). Prevalence of picky eaters among infants and toddlers and their caregivers' decisions about offering a new food. Journal of the American Dietetic Association, 104, s57-s64.

Centers for Disease Control and Prevention. (2014). Prevalence of autism spectrum disorder among children aged 8 years Autism and developmental disabilities monitoring network. 11 Sites. United States, 2010. MMWR Surveillance Summary, 63(SSO2), 1-21.

Cerro, N., Zeunert, S., Simmer, K.N., \& Daniels, L.A. (2002). Eating behaviour of children 1.5-3.5 years born preterm: Parents' perceptions. Journal of Paediatrics and Child Health, 38, 72-78.

Davies, W.H., Satter, E., Berlin, K.S., Sato, A.F., Silverman, A.H., Fischer, E.A., . . \& \& Rudolph, C.D. (2006). Reconceptualizing feeding and feeding disorders in interpersonal context: The case for relational disorder. Journal of Family Psychology, 20, 409-417.
Derogatis, L.R., \& Melisaratos, N. (1983). The Brief Symptom Inventory: An introductory report. Psychological Medicine, 13, 595-605.

Dovey, T.M., Staples, P.A., Gibson, E.L., \& Halford, J.C. (2008). Food neophobia and 'picky/fussy' eating in children: A review. Appetite, 50, 181-193.

Drewett, R.F., Corbett, S.S., \& Wright, C.M. (2006). Physical and emotional development, appetite and body image in adolescents who failed to thrive as infants. Journal of Child Psychology and Psychiatry, 47, 524-531.

Dubois, L., Farmer, A., Girard, M., Peterson, K., \& TatoneTokuda, F. (2007). Problem eating behaviors related to social factors and body weight in preschool children: A longitudinal study. International Journal of Behavioral Nutrition and Physical Activity, 4, 9.

Emond, A., Emmett, P., Steer, C., \& Golding, J. (2010). Feeding symptoms, dietary patterns, and growth in young children with autism spectrum disorders. Pediatrics, 126, e337e342.

Farrow, C., \& Blissett, J. (2006). Maternal cognitions, psychopathologic symptoms, and infant temperament as predictors of early infant feeding problems: A longitudinal study. International Journal of Eating Disorders, 39, 128134.

Ford, T., Goodman, R., \& Meltzer, H. (2003). The British Child and Adolescent Mental Health Survey 1999: The prevalence of DSM-IV disorders. Journal of the American Academy of Child and Adolescent Psychiatry, 42, 1203-1211.

Galloway, A.T., Lee, Y., \& Birch, L.L. (2003). Predictors and consequences of food neophobia and pickiness in young girls. Journal of the American Dietetic Association, 103, 692698.

Goh, D.Y., \& Jacob, A. (2012). Perception of picky eating among children in Singapore and its impact on caregivers: A questionnaire survey. Asia Pacific Family Medicine, $11,5$.

Hafstad, G.S., Abebe, D.S., Torgersen, L., \& von Soest, T. (2013). Picky eating in preschool children: The predictive role of the child's temperament and mother's negative affectivity. Eating Behaviors, 14, 274-277.

Jacobi, C., Schmitz, G., \& Agras, W.S. (2008). Is picky eating an eating disorder? International Journal of Eating Disorders, 41, 626-634. 
Jaddoe, V.W., van Duijn, C.M., Franco, O.H., van der Heijden, A.J., van IJzendoorn, M.H., de Jongste, J.C., . . \& Hofman, A. (2012). The Generation R Study: Design and cohort update 2012. European Journal of Epidemiology, 27, 739756.

Maenner, M.J., Schieve, L.A., Rice, C.E., Cunnif, C., Giarelli, E., Kirby, R.S., ... \& Durkin, M.S. (2013). Frequency and pattern of documented diagnostic features and the age of autism identification. Journal of the American Academy of Child \& Adolescent Psychiatry, 52, 401-413.

Marchi, M., \& Cohen, P. (1990). Early childhood eating behaviors and adolescent eating disorders. Journal of the American Academy of Child and Adolescent Psychiatry, 29, $112-117$.

Mascola, A.J., Bryson, S.W., \& Agras, W.S. (2010). Picky eating during childhood: A longitudinal study to age 11 years. Eating Behaviors, 11, 253-257.

McKee, M.D., Maher, S., Deen, D., \& Blank, A.E. (2010). Counseling to prevent obesity among preschool children: Acceptability of a pilot urban primary care intervention. Annals of Family Medicine, 8, 249-255.

Micali, N., Simonoff, E., Elberling, H., Rask, C.U., Olsen, E.M., \& Skovgaard, A.M. (2011a). Eating patterns in a populationbased sample of children aged 5 to 7 years: Association with psychopathology and parentally perceived impairment. Journal of Developmental and Behavioral Pediatrics, 32, $572-580$.

Micali, N., Simonoff, E., Stahl, D., \& Treasure, J. (2011b). Maternal eating disorders and infant feeding difficulties: Maternal and child mediators in a longitudinal general population study. Journal of Child Psychology and Psychiatry, 52, 800-807.

Nicely, T.A., Lane-Loney, S., Masciulli, E., Hollenbeak, C.S., \& Ornstein, R.M. (2014). Prevalence and characteristics of avoidant/restrictive food intake disorder in a cohort of young patients in day treatment for eating disorders. Journal of Eating Disorders, 2, 21.

Nicholls, D., Christie, D., Randall, L., \& Lask, B. (2001). Selective eating: Symptom, disorder or normal variant. Clinical Child Psychology and Psychiatry, 6, 257-270.

Ooi, Y.P., Rescorla, L., Ang, R.P., Woo, B., \& Fung, D.S. (2011). Identification of autism spectrum disorders using the Child Behavior Checklist in Singapore. Journal of Autism and Developmental Disorders, 41, 1147-1156.

Ozonoff, S., Young, G.S., Steinfeld, M.B., Hill, M.M., Cook, I., Hutman, T., ... \& Sigman, M. (2009). How early do parent concerns predict later autism diagnosis? Journal of Developmental and Behavioral Pediatrics, 30, 367-375.

Ringoot, A.P., Tiemeier, H., Jaddoe, V.W., So, P., Hofman, A., Verhulst, F.C., \& Jansen, P.W. (2015). Parental depression and child well-being: Young children's self-reports helped adressing biases in parent reports. Journal of Clinical Epidemiology, 62, 928-939.

Sharp, W.G., Berry, R.C., McCracken, C., Nuhu, N.N., Marvel, E., Saulnier, C.A., ... \& Jaquess, D.L. (2013). Feeding problems and nutrient intake in children with autism spectrum disorders: A meta-analysis and comprehensive review of the literature. Journal of Autism and Developmental Disorders, 43, 2159-2173.

Sikora, D.M., Hall, T.A., Hartley, S.L., Gerrard-Morris, A.E., \& Cagle, S. (2008). Does parent report of behavior differ across ADOS-G classifications: Analysis of scores from the CBCL and GARS. Journal of Autism and Developmental Disorders, 38, 440-448.

Skeem, J.L., Schubert, C., Odgers, C., Mulvey, E.P., Gardner, W., \& Lidz, C. (2006). Psychiatric symptoms and community violence among high-risk patients: A test of the relationship at the weekly level. Journal of Consulting and Clinical Psychology, 74, 967-979.

So, P., Greaves-Lord, K., van der Ende, J., Verhulst, F.C., Rescorla, L., \& de Nijs, P.F. (2013). Using the Child Behavior Checklist and the Teacher's Report Form for identification of children with autism spectrum disorders. Autism: the International Journal of Research and Practice, 17, 595-607.

Swinbourne, J.M., \& Touyz, S.W. (2007). The co-morbidity of eating disorders and anxiety disorders: A review. European Eating Disorders Review, 15, 253-274.

Tick, N.T., van der Ende, J., \& Verhulst, F.C. (2007). Twentyyear trends in emotional and behavioral problems in Dutch children in a changing society. Acta Psychiatrica Scandinavica, 116, 473-482.

Verhulst, F.C., dervan Ende, J., \& Koot, H.M. (1997). Dutch manual for the Teacher's Report Form (TRF). Rotterdam: Department of Child and Adolescent Psychiatry, Erasmus University Medical Center - Sophia Children's Hospital.

Wright, C.M., Parkinson, K.N., Shipton, D., \& Drewett, R.F. (2007). How do toddler eating problems relate to their eating behavior, food preferences, and growth? Pediatrics, 120, e1069-e1075.

Accepted for publication: 7 December 2015 